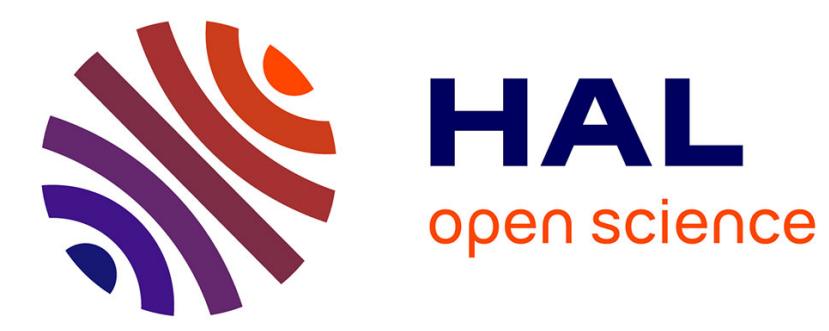

\title{
Situer la possession. Du droit romain de l'appartenance aux nouveaux modèles propriétaires
}

Pierre Thévenin

\section{To cite this version:}

Pierre Thévenin. Situer la possession. Du droit romain de l'appartenance aux nouveaux modèles propriétaires. Clio@Thémis. Revue électronique d'histoire du droit, 2018, 14, pp.En ligne. 10.35562/cliothemis.795 . halshs-02309701

\section{HAL Id: halshs-02309701 \\ https://shs.hal.science/halshs-02309701}

Submitted on 9 Oct 2019

HAL is a multi-disciplinary open access archive for the deposit and dissemination of scientific research documents, whether they are published or not. The documents may come from teaching and research institutions in France or abroad, or from public or private research centers.
L'archive ouverte pluridisciplinaire HAL, est destinée au dépôt et à la diffusion de documents scientifiques de niveau recherche, publiés ou non, émanant des établissements d'enseignement et de recherche français ou étrangers, des laboratoires publics ou privés.

\section{(1) (1) $\$$}

Distributed under a Creative Commons Attribution - NonCommercial - NoDerivatives| 4.0 


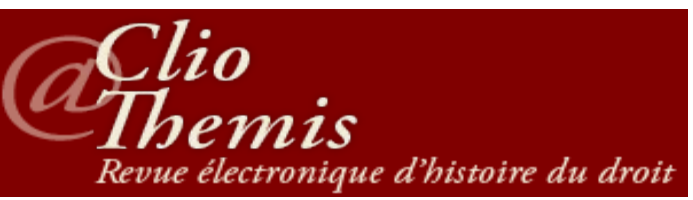

\title{
Situer la possession. Du droit romain de l'appartenance aux nouveaux modèles propriétaires
}

\begin{abstract}
Résumé: En suscitant la recherche de «nouveaux modèles propriétaires », l'évolution contemporaine du droit des biens appelle à revisiter en profondeur l'histoire juridique des formes d'appartenance, pour mettre en lumière l'existence de dynamiques alternatives à celles qui ont soutenu l'émergence de l'idée de propriété exclusive. Le présent article propose d'articuler cet effort d’analyse rétrospective à l'idée de possession, telle que le droit romain l'a dissociée de la propriété, dont Savigny fit l'axiome d'une dogmatique moderne, centrée sur l'idée de personnalité.
\end{abstract}

\begin{abstract}
This article calls for a reengagement with historical forms of legal appropriation. Exploring ways to benefit from a ressource that ran parallel to exclusive property, it aims at providing legal scholars with an archeological touchstone capable of triggering new approaches to property rights. The Roman law of possession, it argues, offers a valuable angle for such an inquiry. Focusing on its interpretation by Savigny, it looks at the part it played in binding classical legal theory with the modern idea of personality.
\end{abstract}

Mots-clés : possession - Savigny - propriété

Key words : possession - Savigny - property

I. Lancés à la recherche de «modèles propriétaires » qui permettraient de mieux refléter l'évolution actuelle du droit des biens, marquée par la multiplication des droits spéciaux ${ }^{1}$, quelques civilistes ont proposé de substituer à la réflexion traditionnellement dévolue au concept de propriété un intérêt plus large pour l'ensemble des « signes » susceptibles d'être interprétés comme une « manifestation » de celle$\mathrm{ci}^{2}$. Cet effort d'élargissement n'est pas sans ombre portée au sein de l'histoire juridique. Au contraire, le regard rétrospectif sur la provenance des catégories du droit civil semble accompagner, comme sa contrepartie solidaire, le travail d'exploration normative auquel s'attachent les auteurs gagnés à cette perspective. Aussi n'est-il pas rare que ceux-ci glissent sua sponte vers le discours de l'Histoire. En rappelant particulièrement l'émergence historique de cette propriété « exclusive » que la tradition des codifications révolutionnaires, confluant à l’article 544 du Code civil, a laissée en partage aux modernités juridiques européennes, ces juristes, civilistes ou comparatistes, s'attachent alors à mettre en perspective, pour mieux la discuter, l'idée de propriété absolue réputée servir de « pilier » au droit civil en vigueur ${ }^{3}$ ou constituer, comme l'avait classiquement entendu Portalis, ce «droit fondamental sur lequel toutes les institutions sociales reposent $\gg^{4}$. Dans ce tableau - ou plutôt ce canevas, le droit d'Ancien Régime trouve naturellement une place de choix. Partie liée à la féodalité, il figure «l'autre » du régime juridique

I Les modèles propriétaires. Actes du colloque international onganisé par le CECOJI - En hommage au professeur HenriJacques Lucas, Paris, Librairie générale de droit et de jurisprudence, 20I2. Cet article est tiré d'une intervention le i8 Novembre 2015 au séminaire «Les signes de la propriété », organisé à l'ENS de Cachan, en partenariat avec l'IDHES (UMR 8533), dont je remercie les organisateurs M. Barbot, M. Cornu, C. Didry et N. Wagener.

2 Voir le séminaire « Les signes de la propriété » mentionné ci-dessus.

3 Pour un remploi récent de cette métaphore traditionnelle, promue par Jean Carbonnier, voir N. Laurent-Bonne, N. Pose, V. Simon, Les piliers du droit civil. Famille, Propriété, Contrat, Paris, Mare et Martin, 2015.

4 J. É. M. Portalis, « Présentation du titre De la propriété devant le Corps législatif », dans P. -A. Fenet, Recueil complet des travaux préparatoires du Code civil, Paris, Videcoq, I836, t. 9, p. 132, cité par F. Zénati, qui fait de la propriété « l'axe ordonnançant [les] dispositions [du code civil français] » (id., «Pour une rénovation de la propriété », Revue trimestrielle de droit civil, 1993 , p. 305-324, p. 305). 
moderne : un droit où non seulement la propriété, divisée, ne présente aucun caractère absolu, mais où elle n'a pas même la valeur d'un foyer particulièrement prépondérant de la réflexion juridique.

2. Avatar technique des « usages politiques du passé » qui retiennent communément l'attention des politistes, des historiens et des sociologues's, ces usages juridiques de l'histoire ont naturellement reçu des formes variées, de la simple annotation introductive ${ }^{6}$ à la référence cardinale 7 en passant par l'argument ponctuel $^{8}$. Ils présentent toutefois un caractère commun: celui d'émaner d'interprètes du droit contemporain, plutôt que d'historiens de métier. Aussi ne s'emploient-ils guère, au premier chef, à reconstituer la réalité des situations juridiques anciennes. S'ils évoquent le souvenir des anciennes modalités de l'appartenance, du domaine divisé ou des droits d'usage collectifs, c'est en manière d'incise, et sans puiser à la méthode historique, proprement entendue comme une appréhension critique des anciennes sources de droit destinée à en restituer la signification et le fonctionnement singuliers 9 . Car le propos de ces usages furtifs de l'histoire est ailleurs. Ils visent à impulser un travail d'élaboration normative, doctrinale sinon législative, tout entier conçu au présent : celui de l'habitat participatif ou des logiciels libres, de la protection des ressources naturelles ou des droits d'auteur collaboratifs ${ }^{10}$.

3. Si l'historien n'apprend sans doute rien de neuf de ces évocations rudimentaires, où résonne l'écho des manuels $s^{\text {II }}$ cette immixtion du passé au cœur du débat juridique contemporain ne peut manquer de l'interpeler. Pour furtives qu'elle soit, l'invocation de l'expérience juridique médiévale, dans un tel contexte, n'est-elle pas pour le surprendre ? À l'heure où se répand la peur que sa discipline périclite ${ }^{12}$, elle apparaît comme une curiosité sympathique, susceptible de le divertir de la tendance à regretter tout à la fois sa «gloire perdue » $^{13}$ et l'époque « où les étudiants rêvaient en latin et les juristes chérissaient le digeste $\gg^{14}$. Si le savoir historique n'a plus peut-être la place qu'il eut jadis au sein des facultés de droit, si la position institutionnelle de l'histoire du droit, en tant que discipline séparée, y devient parfois

$5 \quad$ Voir ainsi, par ordre de disciplines respectives, « Le passé mobilisé », Politix, Revue des sciences sociales du politique, 2, IIo, 2015 ; Les usages politiques du passé, F. Hartog et J. Revel (dir.), Paris, Éditions de EHESS, $200 \mathrm{i}$; « Histoire, mémoire et passé au cour des organisations », Sociologies pratiques, 2, 29, 2014.

6 Ainsi G. Lardeux, «Qu'est-ce que la propriété ? Réponse de la jurisprudence récente éclairée par l'histoire », Revue trimestrielle de droit civil, 2013, p. 74I-758 et J. Rochfeld, « Penser autrement la propriété : la propriété s'oppose-t-elle au communs ? », Revue internationale de droit économique, 3, 28, 2014, p. 351-369.

7 Ainsi du renvoi aux travaux de l'historien du droit Paolo Grossi - lui-même « bien connu des privatistes », comme le relève à juste titre M. Ascheri (id., «Un ordine giuridico medievale per la realtà odierna ? », Rivista trimestrale di diritto e procedura civile, 50, 1996, p. 965-973, p. 965) - chez le comparatiste Ugo Mattei (par exemple id., Beni comuni. Un manifesto, Rome, Laterza, 2olı, p. Io).

8 Ainsi du développement qu'Aurore Chaigneau consacre aux biens communaux sous l'Ancien Régime, en s'appuyant sur un article d'Henri Sée datant de 1923 (id., «Une propriété affectée au commun », dans Fonctions de la propriété et commun. Regards comparatifs, A. Chaigneau (dir.), Paris, Société de législation comparée, 2016, p. 57-70, notamment note Io p. 6o).

9 Pour un rappel vigoureux de la vocation de l'histoire du droit à demeurer une « partie de la science historique », voir M. Stolleis, Rechtsgeschichte schreiben, Bâle, Schwabe, 2008, p. 6.

Io Je cite ici les axes du projet ERC Inclusive mené par Séverine Dusollier, http://cordis.europa.eu/project/rcn/ı92286 en.

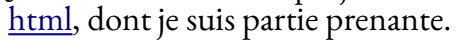

II Judith Rochfeld (voir note 6 ci-dessus) appuie sa référence à la féodalité sur le manuel d'Anne-Marie Patault, dont Philippe Godding remarque qu'il omet de citer les travaux de langue allemande ou anglaise portant sur les structures coutumières qu'il décrit, pourtant communes à l'Europe occidentale (Philippe Godding, « A.-M. Patault, Introduction historique au droit des biens », Cabiers de civilisation médiévale, 35, 139, 1992, p. 263-265). Pour une appréciation nuancée des fortes thèses de Paolo Grossi, souvent prises pour argent comptant par les civilistes et les comparatistes, voir M. Ascheri, op. cit.; E. Conte, «Droit médiéval. Un débat historiographique italien », Annales. Histoire, Sciences Sociales, 57, 6, 2002, p. 1593I6I3 et N. Warembourg, « Peut-on parler d'ordre juridique médiéval ? », Penser l'ordre juridique médiéval et moderne, Regards croisés sur les méthodes des juristes (I), N. Laurent-Bonne et X. Prévost (dir.), Issy-les-Moulineaux, LGDJ, 20I6, p. 23-42.

I2 En 20Io, Jean-Louis Halpérin réprouvait la tendance des historiens du droit, notamment de tradition allemande, à se laisser gagner «par une crainte, peut-être excessive, de voir leur discipline sombrer, même en tant que science auxiliaire » (id., «Le droit et ses histoires », Droit et société, 75, 2, 2010, p. 295-313, p. 295).

I3 J. Whitman, « Bring back the glory! », Rechtsgeschichte. Zeitschrift des Max Planck Institut für europaïsche Rechtsgeschite, 4, 2004, p. 74-8I.

I4 L. Waelkens, « Hans Erich Troje, Crisis digestorum », Tijdschrift voor rechtsgeschiedenis, 8I, 20I3, p. 74-8I. 
« précaire » ${ }^{\mathrm{IS}}$, du moins la figure du passé n’a pas cessé de s’inviter dans la fabrique du droit contemporain. À contre-courant de la tendance contemporaine à délester la théorie du droit de toute inscription dans l'espace et le temps, le simple fait que des auteurs de doctrine soient portés à invoquer l'expérience juridique médiévale revêt un caractère insolite ${ }^{16}$.

4. Dans cet article, l'idée de possession sert de point d'appui, pour jeter les jalons d'une première exploration de ce couloir temporel inattendu, spontanément apparu au sein de la théorie contemporaine du droit des biens. Plusieurs raisons recommandent ce point d'entrée. Tout d'abord, l'importance de l'institution de la possession, dans l'ancien droit privé, peut permettre à l'historien de prolonger, en la complétant peut-être, la référence contemporaine aux anciennes modalités de l'appropriation, pour révéler la physionomie particulière que la pratique et la réflexion juridique avaient donnée au fil des siècles à cette institution, avant que les idées nouvelles ne précipitent l'effacement juridictionnel ou l'abolition législative des droits féodaux. Ensuite cette enquête promet de jeter les bases d'un questionnement plus théorique, pour déterminer d'une part si la possession est effectivement susceptible de constituer l'un des signes de la propriété que nous sommes invités à concevoir aujourd'hui, d'autre part si elle peut effectivement favoriser le contournement doctrinal de l'idée moderne d'une propriété exclusive.

5. Pour rassembler quelques éléments de réponse à cette combinaison de questions, le présent article se propose de lever les obstacles qui gênent la perception contemporaine d'une distinction nette entre la possession et la propriété ( $\$ 6$-Io), en rappelant l'origine romaine de cette distinction ( $\$ \$$ II-I 6 ), puis l'interprétation qu'en a proposée le fondateur de l'école historique allemande, Friedrich Carl von Savigny (\$\$ 17-25). Il cherche enfin à tirer de ces éléments le ressort d'une « histoire juridique de l'accès », susceptible d'enrichir la discussion contemporaine sur l'évolution du droit des biens (\$\$26-28).

\section{L'incertaine distinction de la possession et de la propriété}

6. L’originalité de l'idée de possession n'a rien d'évident. Faut-il tenir la possession pour une modalité, une modification, une suite ou une division de la propriété ? Ou bien le mot de possession qualifie-t-il une situation distincte, incommensurable au droit de la propriété au point même qu'il soit difficile de la tenir pour un «signe » de celle-ci ? Cette alternative simple appelle naturellement une très large série de considérations de droit positif, qui toucheront par exemple à l'examen des preuves de la propriété des immeubles ${ }^{17}$ et à la question des objets de la possession, notamment la question de savoir s'il est possible de posséder un bien incorporel, comme un droit d'auteur ${ }^{18}$. Pourtant, plusieurs éléments concourent à faire obstacle à la construction d'une telle comparaison. Une tendance culturelle passablement diffuse pousse en effet à faire de la possession et de la propriété deux figures contigües d'une seule et même relation fondamentale d'appartenance. Cette tendance s'alimente au moins à trois sources, concurrentes quoique fort distinctes entre elles.

Is «The institutional position [of the discipline of legal history] at the law departments has become precarious », Th. Duve, «German Legal History: National Traditions and Transnational Perspectives », Rechtsgeschichte - Legal History, 22, 2014, p. 16-48.

I6 À mesure que les sources de droit locales, nationales et transnationales entrent dans des rapports d'interférence complexes, le droit serait appelé à former un « archipel » de règles disparates (W. Twinning, General Jurisprudence. Understanding Law from a Global Perspective, Cambridge, Cambridge University Press, 2009, p. 67), de moins en moins clairement « situables » dans le temps et l'espace (N. Walker, « Out of place and out of time : law's fading coordinates », Edinburgh School of Law Working Paper Series, 2009).

I7 Sur la possession comme fondement d'une présomption de propriété immobilière, voir W. Dross, « Qui doit être déclaré propriétaire lorsque aucun des litigants ne l'est ? : le conflit du titre a non domino et de la possession sans animus domino, Civ. $3^{\mathrm{e}}, 8$ oct. 2015, n' ${ }^{\circ}$ 14-16.963 », Revue trimestrielle de droit civil, 2016, p. 154-156.

I8 A. Pélissier, Possession et meubles incorporels, Paris, Dalloz, 20or ; B. Parance, La possession des biens incorporels, Paris, Librairie générale de droit et de jurisprudence, 2008. 
7. Elle procède d'abord de l'assimilation philosophique et générale, qui regarde l'institution de la propriété comme le produit dérivé d'une occupation originaire des choses. Selon le trope emblématique du jusnaturalisme classique, la terre fit, aux commencements de la société humaine, l'objet d'une prise de possession, qui marqua les prémisses de la propriété. Chez Grotius, cette prise de possession est collective, et se présente sous une double espèce : celle, expresse, d'un partage des biens aux temps archaïques, par où «l'on partagea les choses qui étaient auparavant en commun » et celle, tacite, d'une occupation de la terre. L'institution civile de la propriété repose donc sur l'événement ancestral, soit d'un partage explicite des terres par la communauté, soit sur la décision collective de laisser les individus occuper une portion de terre pour eux-mêmes.

Simulatque enim communio displicuit, nec instituta est divisio, censeri debet inter omnes convenisse, ut quod quisque occupasset id proprium haberet.

Car du moment qu'on ne voulut plus laisser les choses en commun, tous les hommes furent censés et dûrent être censés avoir consenti, que chacun s'appropriât, par droit de premier occupant, ce qui n’aurait pas été partagét9.

8. Par extrapolation du droit romain de l'occupation - que le Corpus iuris civilis ne mentionnait guère qu'au titre, fort étroit, de la capture des bêtes sauvages ${ }^{20}$, Grotius mettait ainsi sur pied une philosophie naturelle de la propriété. Celle-ci devait contribuer à aligner l'une sur l'autre les idées de possession et de propriété. Le développement de ce que les historiens des idées politiques nomment individualisme possessif ${ }^{21}$ vint accentuer cette tendance. En qualifiant de $\left\langle\right.$ chimère $\gg^{22}$ le renvoi à un partage primitif de la terre, Barbeyrac et Pufendorf resserrèrent ainsi l'analyse autour de la seule figure «moderne » de l'occupation, renforçant davantage encore l'indexation de la propriété à la prise de possession. Cependant une autre ligne de pensée, courant depuis Locke, poussait à présenter la possession comme un fait anthropologique, commençant par la possession du corps propre pour s'étendre aux choses mêmes ${ }^{23}$. L'un dans l'autre, la pensée du droit naturel imprègne encore largement le sens commun, qui tend lui aussi à amalgamer les concepts de possession et de propriété, sans inciter à plus de prudence dans la distinction de ces termes - une distinction qui ne peut jamais constituer qu'un artifice de droit positif, dépourvu de signification « philosophique » réelle ${ }^{24}$.

9. Parallèlement à cette première tendance, certains développements propres à la réflexion économique tendent aujourd'hui à subordonner l'analyse des institutions juridiques à l'évaluation des préconditions de la croissance économique ou du développement d'un marché capitaliste. Liée à la perspective d'une mesure de la performance économique des institutions ${ }^{25}$, cette école de pensée conduit à effacer la figure de la possession, au profit d'une comparaison entre différentes espèces de propriété. Dans le domaine des études rurales, par exemple, la question est de savoir si l'institution de la propriété parfaite a constitué un

I9 Hugonis Grotii De jure belli ac pacis libri très : in quibus jus naturae et gentium, item juris publici praecipua explicantur, Rotterdam, Arnoldum Leers, I680, II, 2, 4 ; trad. J. Barbeyrac, Le droit de la guerre et de la paix, Amsterdam, Pierre de Coup, 1724 ; trad. P. Pradier-Fodéré, Paris, Presses universitaires de France, 1999 [1867].

20 «Tous les animaux qui sont capturés (capiuntur) sur terre, dans la mer et dans le ciel, c'est-à-dire les bêtes sauvages, les oiseaux et les poissons, sont à ceux qui les capturent (capientium fiunt) », D. 4I, I, I, I, et pour l'analyse de ce texte romain par Grotius, De jure belli, op. cit., II, 8, 2, trad. P. Pradier-Fodéré, p. 286. Voir également A.-J. Arnaud, « Réflexions sur l'occupation, du droit romain classique au droit moderne », Revue historique de droit français et étranger, 46, 1968, p. I832Io, qui rappelle à juste titre que le mot occupatio n'apparaît pas en droit romain. Ajoutons qu'on ne trouve pas d'entrée « occupatio » au dictionnaire d’Albéric de Rosate, Dictionarium iuris tam civilis quam canonici, Venise, I58I.

2I C. B. Macpherson, The Political Theory of Possessive Individualism: From Hobbes to Locke, Oxford, Oxford University Press, 1962, trad. fr. M. Fuchs, Paris, Gallimard, 1971.

22 C'est le terme de Barbeyrac, dans sa note i2 à sa traduction commentée de Grotius, II, 2, 3, op. cit.

23 R. Castel, C. Haroche, Propriété privée, propriété sociale, propriété de soi. Entretiens sur la construction de l'individu moderne, Paris, Fayard, 20oI.

24 L'étude fouillée que Céline Spector consacre à la propriété chez Montesquieu ne mentionne pas la possession, à laquelle la grande Encyclopédie consacre pourtant un chapitre de droit positif (id., «Variations de la propriété : Montesquieu contre l'individualisme possessif », dans Inventions et critiques du libéralisme. Le pouvoir, la personne, la propriété, B. Bachofen (dir.), Lyon, ENS éditions, collection « Theoria », 2008, p. 95-II6). À l'inverse, le sens juridique du terme n'affleure guère de la « philosophie des possessions » proposée par Didier Debaise (id., Philosophie des possessions, Paris, Les Presses du Réel, 2oII, malgré le sort que Bruno Latour fait à cette signification dans sa contribution au même volume).

25 Voir K. E. Davis et M. B. Kruse, « Taking the measure of Law. The case of the Doing business project », Law and Social Inquiry, 32, 4, 2007, p. 1095-III9. 
facteur déterminant de la croissance de la production agricole. Un tel débat s'organise alors autour de l'alternative binaire entre la propriété divisée des dominia médiévaux d'un côté, et la propriété parfaite des codifications napoléoniennes de l'autre - la question étant de mesurer l'efficacité économique relative, pour la production et la croissance agricole, de ces deux variétés d'organisation de la propriété. Il s'ensuit naturellement une certaine éclipse de la question des situations possessoires, dont la spécificité juridique tend dès lors à se trouver occultée ${ }^{26}$.

Io. Enfin c'est l'actualité juridique elle-même qui tend à rapprocher la possession de la propriété. En son article 9, la loi n ${ }^{\circ} 2015-177$ du 16 févr. 2015 a en effet procédé à l'abrogation des actions possessoires ${ }^{27}$. Cette évolution, il est vrai, était annoncée. À deux reprises, la Cour de cassation avait déjà demandé la suppression des dispositions du Code civil relatives aux actions possessoires ${ }^{28}$. Les juges estimaient que ces procédures devaient être écartées du droit positif. Ils reprenaient ce faisant une doléance formulée par plusieurs juristes, en premier lieu la Proposition de réforme $d u$ Livre II $d u$ Code civil relatif aux biens, que l'Association Henri Capitant des amis de la culture juridique française avait avancée en $2006^{29}$. Cette proposition recommandait de redéfinir la notion de la possession, pour l'identifier à une modalité de la propriété. Alors que la notion n'est traitée qu'à la fin du livre III du Code civil, sous son aspect de prescription, comme une manière d'acquérir la propriété, il s'agissait de regrouper dans un titre unique aussi bien la propriété que la possession.

\section{Attaches généalogiques de cette dissociation}

II. Ainsi donc, il n'y a rien d'évident à vouloir comparer les idées de possession et de propriété : le jusnaturalisme philosophique, les études économiques de veine institutionnaliste et la doctrine civiliste elle-même semblent aujourd'hui s'accorder, non pas certes à les identifier, mais à les placer dans un rapport de continuité. Un exercice de généalogie juridique - approfondissant en quelque sorte le sillon déjà tracé par cette partie de la doctrine contemporaine qui se plaît à évoquer le spectre des configurations juridiques médiévales, permet pourtant de justifier, sinon le maintien d'une distinction nette entre les notions de possession et de propriété - désormais désavouée par le législateur, du moins le souci de les comparer soigneusement, au moyen de l'histoire, sans trop se hâter de les assimiler l'une à l'autre.

I2. C’est vers Rome que cette généalogie conduit, et ceci par deux voies différentes. D’un point de vue strictement historique, elle renvoie d'abord au développement même de l'idée juridique de possessio, que la lente construction de l'Italie romaine, du $\mathrm{IV}^{\mathrm{e}}$ au ${ }^{\mathrm{er}}$ siècle avant J.-C., a distinguée d'avec la propriété quiritaire. Le mot de possessio désignait alors avant tout la situation des occupants d'une portion de l'ager publicus, c'est-à-dire des terres agricoles gagnées par les conquêtes progressives de l'Italie, dont l'État était tenu pour l'unique propriétaire. Inversement la propriété quiritaire (dominium ex iure Quiritium) était réservée aux propriétaires d’un bien-fonds situé sur l’ager romanus. De la propriété à la possession, il y a donc la même différence qu'entre un espace appropriable par les individus, ponctionné sur celui de la cité,

26 Ainsi la figure de la possession n'apparaît-elle pas dans l'ouvrage collectif d'histoire économique Property Rights, Land Markets and Economic Growth in the European Countryside (Thirteenth-Twentieth Centuries), G. Béaur et $\mathrm{Ph}$. Schofield (dir.), Turnhout, Brepols, 20I3. Pour une introduction aux recoupements de ces analyses économiques avec l'histoire des institutions juridiques anciennes, voir M. Barbot, « When the History of Property Rights Encounters the Economics of Convention. Some Open Questions Starting from European History », Historical Social Research, I5I, 40, 20I5, p. 78-93.

27 N. Cayrol, « Abrogation des actions possessoires », Revue trimestrielle de droit civil, 2015, p. 705-7II.

28 Rapport annuel 2009 de la Cour de cassation - Les personnes vulnérables dans la jurisprudence de la Cour de cassation, Paris, 2009, I7 ; Rapport annuel 2010 de la Cour de cassation - Le droit de savoir, Paris, 20I0, I3.

29 Association Henri Capitant des amis de la culture juridique française, Proposition de réforme du Livre II du Code civil relatif aux biens, Paris, 2006, 8. Voir de même J.-L. Bergel, « Protection de la possession : faut-il maintenir une protection spécifique? », Droit et patrimoine, 2013, p. I7. 
et un espace dont la propriété est réservée à l'État, coïncidant avec les terres fraîchement conquises sur l'ennemi ${ }^{30}$.

I3. En tout état de cause, il est certain que l'encadrement des situations possessoires est né de la spécificité juridique de l'ager publicus, que les citoyens romains, anciens soldats ou colons, exploitaient de fait, sans pouvoir en être propriétaires. Comme le résume Fustel de Coulanges, à propos de la Gaule des deux premiers siècles de l'Empire, où la distinction fut la plus vigoureuse, «le propriétaire du sol était l'Empereur, les hommes n'y étaient que possesseurs $\gg^{31}$. Une différence formelle, essentiellement procédurale, s'est alors attachée à cette situation, offrant une deuxième raison d'aborder par le droit romain l'histoire juridique de la possession. En suscitant la formulation d'un régime d'appartenance distinct de celui de la propriété, le droit romain de la possession a acquis la valeur d'une sorte de paradigme, marquant non seulement l'histoire juridique de Rome, mais l'ensemble des interprétations qui se sont greffées aux sources des compilations justiniennes, pour construire l'idée moderne de possession. Cela engage à considérer non pas tant le droit romain lui-même, que le système moderne de ses interprétations, rassemblé dans ce que le fondateur de l'école historique du droit nommait par anachronisme le «droit romain actuel ». Cette expression, on le sait, est celle du fondateur de l'école historique du droit en Allemagne, Friedrich Carl von Savigny ${ }^{32}$. Elle désignait le système ordonné des interprétations savantes des sources du corpus iuris civilis, où le mot de « système » prend une coloration particulière, à la fois d'ensemble ordonné des idées juridiques, et de science totale, entendue au sens philosophique qui traverse le développement de l'Université allemande au cours du XIX siècle spécialement à Berlin où triompha l'idéal humboldtien de liberté académique, et où Savigny inaugura la chaire de droit romain en $\mathrm{I}^{8} \mathrm{IO}^{33}$.

I4. Avant d'aborder cette littérature particulière, dont l'influence fut considérable, il sera bon de préciser les raisons qui la rendent pertinente pour aborder l'histoire des signes de la propriété. Ces raisons sont au nombre de trois. La première tient à l'originalité de ce système de droit savant, dont Savigny fut le premier promoteur. On a appelé ce système celui du Juristenrecht, du droit construit par les juristes - c'est-à-dire par les juristes universitaires. Cela ne signifiait pas, ou pas seulement, que cette doctrine avait des allures particulièrement intellectuelles et érudites, mais renvoyait plutôt au type de répercussion qu'elle a pu avoir sur la pratique. En effet, le statut des professeurs de droit prévoyait qu'ils siègent comme magistrats des cours d’appel. Cela donnait à l'étude du droit romain la dimension d'une « mission », pour reprendre un terme employé par Savigny dans ses écrits programmatiques, une mission qui ne concernait pas seulement l'enseignement, l'érudition ou ce que nous appellerions la doctrine, mais bien la configuration du droit positif de l'époque, que les professeurs observaient et contrôlaient directement ${ }^{34}$.

15. Or c'est précisément de ce point de vue que la question de la possession a reçu une importance stratégique. Elle offrait un préalable indispensable à l'édification du «droit romain actuel ». Savigny consacre sa première monographie au Droit romain de la possession, en I803, un an avant la promulgation

30 B. G. Niebuhr, Römische Geschichte, Berlin, I853, Reimer, t. 2, p. 436, trad. Histoire romaine, t. 3, Paris, F.-G. Levrault, I830, p. 200 ; Fr. Bozza, «Sull'origine del possesso », Annali Macerata, 6, 1930, p. I89 sq. ; id., La possessio dell'ager publicus, 2 t. Milan, Giuffrè, 1938 ; Cl. Nicolet, Les Gracques ou crise agraire et révolution à Rome, Paris, Julliard, 1967, p. 98 passim; L. Capogrossi Colognesi, La struttura della proprietà e la formazione dei « iura praedorium» nell'età repubblicana, Milan, Giuffrè, 1969 .

3I F. de Coulanges, Les origines du régime féodal. Le bénéfice et le patronat, Paris, C. Julian, 1890, p. 65 sq.

32 F. C. von Savigny, System des heutigen römischen Rechts, 8 t., Berlin, Veit und Comp., I840-I849; trad. fr. Ch. Guenoux, Traité de droit romain, 8 t., Paris, Firmin Didot Frères, I840-1851.

33 W. Rüegg (éd.), Geschichte der Universität in Europa, vol. 3, Vom 19. Jahrundert zum Zweiten Weltkrieg (I80o-1945), Beck, Münich, 2004. Sur les accointances philosophiques de Savigny, il existe une importante littérature, parmi laquelle il faut citer J. Rückert, Idealismus. Jurisprudenz und Politik bei F. C. von Savigny, Edelsbach, 1984; D. Nörr, Savignys philosophische Lehrjabre. Ein Versuch, Francfort-sur-le-Main, Klostermann, I994; O. Jouanjan, «Un tournant philologique dans la science du droit. Histoire et métaphysique chez Savigny », dans Études en l'honneur de Gérard Timsit, Bruxelles, Bruylant, 2004, p. I07-125; P. Thévenin, Le monde sur mesure. Une archéologie juridique des faits, Paris, Classiques Garnier, 2017.

34 F. C. von Savigny, «Vom Beruf unsrer Zeit für Gesetzgebung und Rechtswissenschaft », dans J. Stern, Thibaut und Savigny. Ein Programmatischer Rechtsstreit auf Grund ibrer Schriften, Berlin, Vahlen, 1914, p. 72-86; trad. fr. A. Dufour, De la vocation de notre temps pour la jurisprudence, Paris, Presses universitaires de France, 2006. 
du code napoléonien et deux ans avant la bataille d'Iéna ${ }^{35}$. Ce traité ne deviendra pas seulement, pour tout le $\mathrm{XIX}^{e}$ siècle, un modèle de méthode d'interprétation « actuelle» des sources romaines - ni même l'image, pour le dire avec John Austin, « du plus abouti et du plus maîtrisé de tous les livres qui traitent de droit ${ }^{36}$. Comme l'a bien montré James Whitman, il était également en prise sur la question agraire qui constituait l'un des premiers problèmes politiques qui se posaient à l'Allemagne de la première moitié du $\mathrm{XIX}^{\mathrm{e}}$ siècle, où la plupart des paysans continuaient en effet d'être soumis à un régime juridique de type féodal ${ }^{37}$. Sous des formes qui variaient beaucoup selon les principautés et les États, cette situation perdura au moins jusqu'à l'émancipation de I848, la Bauernbefreiung. C'est dans ce contexte que paraît en 1803 l'ouvrage qui allait faire la gloire du jeune Savigny.

I6. Une dernière raison de partir du traité de Savigny est de nature dogmatique. C'est que son interprétation, à rebours des tendances diffuses et convergentes que nous venons d'indiquer, durcit autant qu'il est possible la distinction de la possession d'avec la propriété. Elle offre donc à l'historien de la pensée juridique un levier de connaissance remarquable, pour engager la discussion de savoir jusqu'à quel point la possession est susceptible d'apparaître comme l'un des signes qui « font la propriété ». En prenant le Traité de la possession en droit romain de Savigny, publié en I803, comme fil conducteur, il s'agira d'atteindre un double objectif, premièrement, de présenter un modèle d'interprétation de la possession établissant nettement sa distinction d'avec la propriété, deuxièmement, d'examiner l'intérêt rétrospectif que peut présenter cette interprétation, dans le contexte de la remise en cause contemporaine du concept absolu de la propriété.

\section{L'interprétation du droit romain de la possession chez Savigny}

17. Dans les sources du droit justinien, le rapport formel de la possession à la propriété se présente sous deux points de vue distincts. La propriété apparât d'abord comme une conséquence possible de la possession d'un bien, qui lorsqu'elle est de bonne foi et continuée pendant une période donnée - trente ans le plus souvent pour les immeubles, fait acquérir un droit de propriété, par prescription acquisitive. Sous certaines conditions, la possession constitue en ce sens la prémisse d'une acquisition possible de la propriété. Ce lien est encore explicite lorsque la prise de possession s'applique à une chose sans maître, c'est-à-dire à une chose qui n'appartient à personne - comme un animal sauvage ou une terre tombée en déréliction, il s'agit alors de l'occupatio ${ }^{38}$; ou encore lorsque la prise de possession vient réaliser l'élément corporel d'un transfert de propriété (traditio) ${ }^{39}$. Cependant la possession fait également l'objet d'une protection indépendante de la propriété. Le prêteur la protège en effet par un certain nombre d'interdits, dont l'octroi ne repose pas, du moins d’après Savigny, sur la considération de la propriété, actuelle ou escomptée à titre d’effet futur de l'usucapio.

I8. La présentation des interdits prétoriens sera utile à l'explication de ce point. En reprenant une classification que les juristes médiévaux ont établie sur la base d'un commentaire de Paul à l'édit du préteur $^{40}$, on peut dire que les interdits possessoires romains se divisent en trois classes, selon la nature du

35 F. C. von Savigny, Das Recht des Besitzes, réédition de la $7^{\mathrm{e}}$ édition revue et augmentée par Adolf R. Rudorff, Scientia Verlag, Aalen, 1990 (désormais noté $R B$ ), trad. fr. J. Beving d'après la $6^{e}$ édition de 1837 , Traité de la possession d'après les principes du droit romain, Bruxelles, Société belge de librairie, I840; trad. Ch. Faivre d’Audelange, d'après la $6^{\mathrm{e}}$ édition de I837, Paris, Joubert, I845. Sur cet ouvrage, voir K. Moriya, Savignys Gedanke im Recht des Besitzes, Francfort-sur-le-Main, Klostermann, 2003.

36 «Of all books upon law the most consummate and masterly », cite par H. Kantorowicz, « Savigny and the historical school of law », dans Rechtshistorische Schriften, C. F. Müller, Karlsruhe, 1970, p. 419-434.

37 J. Q. Whitman, The Legacy of Roman Law in the German Romantic Era, Princeton, Princeton University Press, 1990.

38 Voir note 22 ci-dessus.

39 Voir le titre De adquirendo rerum dominio : des manières d'acquérir la propriété, D. 4I.I.

40 D. 43. I. 2. 3. Cette présentation est relayée par le droit romano-canonique. Je suis ici la classification qui se trouve dans une glose d'Innocent IV au décret de Gratien: «triplex est iudicium possessorium, vel pro adipiscenda possessione, vel 
but auquel ils visent. Ils protègent d'abord l'entrée en possession (pro adipiscenda possessione). Ainsi l'interdit quorum bonorum ${ }^{41}$ est donné au bonorum possessor, c'est-à-dire à celui qui a hérité d'un patrimoine par la voie prétorienne ( $a b$ intestat). Il oblige les héritiers légitimes, les heredes sui appelés à l'hérédité par le droit civil, à laisser le bonorum possessor entrer en possession des biens que la succession prétorienne lui a laissés en partage. Les interdits peuvent également viser à remettre en selle le possesseur (pro recuperanda possessione), soit lorsqu'il a été éjecté par la force de sa possession (interdit unde vi), soit lorsqu'on s'est emparé subrepticement de celle-ci, en son absence, clandestinement ou par ruse (interdit $v i$ aut clam). Enfin, les interdits peuvent garantir le possesseur contre la simple menace d'un trouble (pro retinenda possessionis), soit en matière d'immeubles (interdit uti possidetis), soit en matière de meubles (interdit utrubi).

19. Tout le traité de Savigny va donc consister à expliquer le fondement de ces différents interdits. La première affirmation de Savigny, paradoxale et suggestive, est que l'interdit possessoire n'est jamais donné au possesseur en raison d'un « droit » qu'il aurait sur la chose.

Da der Besitz an sich kein Rechtsverhältnis ist, so ist auch die Störung desselben keine Rechtsverletzung.

De même qu'en soi la possession n'est pas un rapport de droit, le trouble de la possession n'est pas la violation d'un droit ${ }^{42}$.

Il faut donc supposer aux interdits un autre fondement que la possession en tant que telle, qui n'est pas un rapport de droit mais un «simple fait » (blosses Faktum) ${ }^{43}$.

20. En vérité le refus d’assimiler le trouble de la possession à la violation d'un droit tire sa vraisemblance du parallélisme entre deux moyens de droit : les interdits possessoires de droit prétorien et l'action pétitoire, en particulier la revendication (vindicatio rei). À la différence des interdits qui constituent des moyens extraordinaires, l'action en revendication représente l'actio in rem par excellence et une action ordinaire de droit civil proprement dit ${ }^{44}$. En déniant que le trouble de la possession relève d'aucune contravention à une règle de droit (Rechtsverletzung), Savigny ne fait donc que souligner le contraste qui oppose les interdits à l'action en revendication. Comme toute action civile, celle-ci repose sur une formule pétitoire (formula petitoria), par laquelle le plaignant fait valoir que tel de ses droits a été violé. L'editio actionis indique alors à quelle action il choisit de recourir pour demander réparation de cette violation, de manière à rétablir le droit réel qu'il a sur la chose ${ }^{45}$.

2I. Dans ce cas, l'affrontement des parties se traduit par une concurrence des preuves de la propriété : chacun des adversaires doit établir l'authenticité de son titre. Cela suppose de remonter dans le temps, pour montrer que le titre dont on se prévaut est autorisé, le cas échéant, par un second titre, plus ancien que celui de l'autre. Il faudra prouver non seulement qu'on a reçu la chose en héritage, par exemple, mais que le défunt dont elle a été héritée avait lui-même sur elle un titre de propriété authentique ; ou bien encore que le vendeur dont on a acquis la chose était lui-même propriétaire de bonne foi, et ainsi de suite. Pour établir son droit « il fallait régresser jusqu'au titre le plus ancien, et pour cela combattre des titres rivaux, s'engager [...] dans l'histoire chaotique des héritages, des partages, des aliénations et des

recuperanda, vel retinenda $\gg$ (Glose de recuperanda à c. pastoralis, de causa possessionis e proprietatis (X, 2, I2, 5), dans Decretum Gratiani emendatum et notationibus illustratum una cum glossis Gregorii XIII pont. max. iussu editum, Venise, I595). Savigny rejette au contraire l'idée que les interdits pro adipiscenda possessione soient véritablement possessoires ( $R B$, \$35, p. 384-385).

4I Cet interdit fait l'objet du titre Quorum bonorum du Digeste (D. 43.2).

$42 R B, \$ 2$, p. 30 .

43 «Il est clair que la possession, par elle-même et selon son concept originel, est un simple fait (dass der Besitz an sich, seinem ursprünglichen Begriffe nach, ein blosses Faktum ist) $\gg, R B, \S 5$, p. 43.

44 W. Buckland, A Manual of Roman Private Law, Cambridge, Cambridge University Press, 1947 [1925], ch. 16, « Actions, classifications, judicial systems ».

45 Je suis la définition que Placentin retient de l'editio actionis, comme choix d'une forma petendi (Placentinus in librum septimum codicis, Lyon, 1536 , ad C. 2, 1,3 ). 
cessions $\gg^{46}$. En pratique la réunion d'une telle preuve s'avérait si ardue, que les médiévaux comme on sait l'ont nommée diabolique : probatio diabolica.

22. En offrant une alternative à cette régression indéfinie, les interdits possessoires viennent parer à cette difficulté. Ils permettent de suspendre ou de repousser la procédure en revendication, c'est-à-dire la procédure qui porte sur le droit véritable. Avant même de trancher la question légale de la propriété ou de décider laquelle des deux parties fait valoir le titre le plus solide, le possesseur se voit offrir la possibilité de réclamer des interdits qui lui assurent d'être protégé dans la jouissance de sa possession. Les interdits le prémunissent en effet, sans autre examen du fond de son droit, contre toute violence qui viserait à l'empêcher d'entrer en possession, à le déloger de son siège ou à lui bloquer l'accès de sa possession après l'avoir occupée en son absence et clandestinement. La formule par laquelle le préteur introduit l'interdit unde vi témoigne du caractère provisoire de cette procédure extraordinaire :

Ait praetor : Unde tu illum vi dejecisti : de eo, quaeque ille tunc ibi habuit, tantummodo intra annum judicium dabo.

Le préteur dit : «si tu as expulsé violemment quelqu'un de sa possession, je donnerai une action [en justice], seulement dans l’année, à raison de ce que celui [que tu as dépossédé] avait dans cet endroit ${ }^{47}$.

23. Si le préteur accorde l'interdit au possesseur, c'est donc sur la base d'une simple appréciation des faits. Pour donner lieu à cette procédure, il suffit de constater qu'une personne a été délogée de sa possession avec violence. La condition de l'interdit, nécessaire et suffisante, est de l'ordre d'un constat de fait : « unde tu... judicium dabo ». Ici il est fait abstraction non seulement du droit de la victime à posséder la chose, mais du droit que le fauteur de trouble, autrement dit le responsable de l'expulsion, revendique éventuellement sur celle-ci. C'est encore le sens des actions possessoires du Code civil, dont l'article 2282 établit que « la possession est protégée sans avoir égard au fond du droit, contre le trouble qui l’affecte ou la menace ». Comme l'écrit Yan Thomas, assimilant les interdits à des expédients « valant en marge de la régularité normative » : « chacun est pour un temps frustré du droit auquel il prétend, sans être contraint d'y devoir jamais renoncer $\gg^{48}$. On comprend en quel sens le recours à l'interdit possessoire fait figure de moyen de droit parallèle à la revendication d'un titre légal. Il conforte provisoirement la situation du possesseur, en l'attente de tout éclaircissement éventuel du droit qui est en jeu sur le fond. En ce sens, comme le posent plusieurs passages de Paul et d'Ulpien, les interdits procèdent davantage du pouvoir de commander, qui ne se règle pas sur les principes, que de la juridiction, qui énonce le droit une fois pour toutes ${ }^{49}$.

24. Tout en partant de ces réalités procédurales romaines, Savigny va accentuer le sens théorique de leur différence. En assimilant la possession à un simple fait, c'est-à-dire à une détention, il va fonder la protection juridique de la possession sur l'association des interdits à des faits de violence :

Wenn nun die Störung des Besitzes gewaltsam geschieht, so liegt in dieser Störung eine Rechtsverletzung, weil jede Gewaltthätigkeit unrechtlich ist, und dieses Unrecht ist es, was durch ein Interdict aufgehoben werden soll. Si maintenant le trouble de la possession s'accomplit violemment, il y a là une violation du droit, parce que tout acte de violence est inique, et c'est cette iniquité qu'on réprime par un interdit ${ }^{50}$.

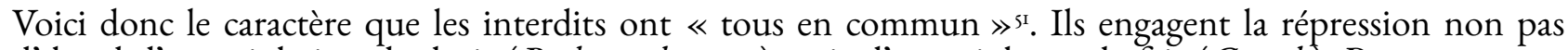
d'abord d'une violation de droit (Rechtsverletzung) mais d'une violence de fait (Gewalt). Parce que tout acte de violence est réputé inique (unrecht), les interdits sont accordés aux possesseurs, quoiqu'ils n'aient aucun droit à faire valoir sur la chose qu'ils possèdent.

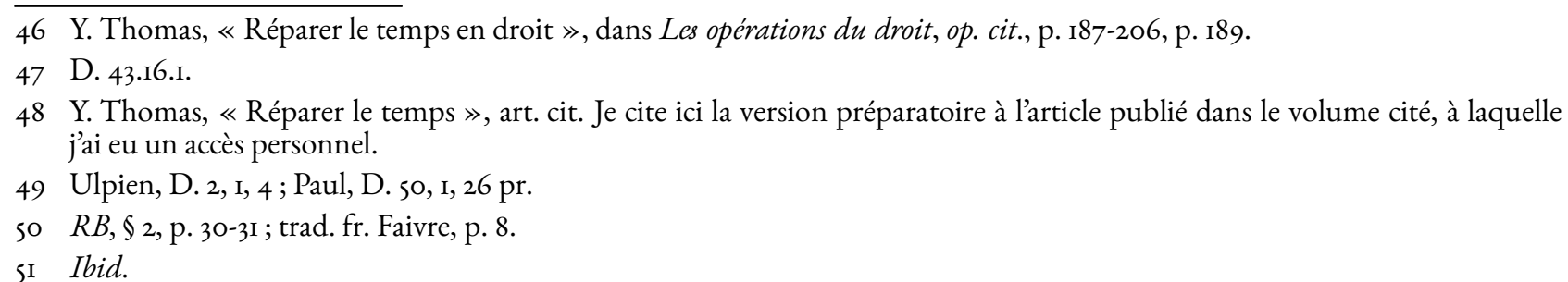


25. C'est donc l'iniquité de la violence, considérée dans sa simple forme, qui représente aux yeux de Savigny le véritable fondement des interdits. Ceux-ci ne protègent pas le droit du possesseur, mais ils répriment l'offense qui résulte de la violence dont il a fait l'objet. Au plan technique ils s'analysent non pas du tout comme des formes imparfaites ou provisoires de revendication, mais comme un équivalent prétorien des actions ex delicto ${ }^{52}$. Même lorsqu'ils n'impliquent aucun acte de violence avéré, comme dans le cas couvert par l'interdit unde vi ou l'interdit utrubis', du moins la crainte d'une violence future, envisagée par la clausule vim fieri veto qui s'adjoint à divers interdits, notamment l'interdit uti possidetiss4, suffit à établir ce lien. Comme il est évident, la solution offerte par Savigny conduit à renverser la longue tradition de l'usus modernus pandectarum, qui rangeait la possession parmi les droits réels (jura in re), à côté de la propriété, de l'hypothèque, de la servitude et de l'héréditéss. Si la possession fait l'objet d'une protection, celle-ci se fonde sur le droit de la personnalité, ou encore sur la dignité de la personne, qui n'a pas à subir l'injustice formelle d'être violentée. En ce sens non seulement les interdits ne sont pas « des revendications provisoires instituées pour régler le procès sur la propriété », comme Jhering proposera de l'admettre, mais ils n'encadrent aucun rapport à la chose qui serait de l'ordre d'un « segment » de jouissance ou d'usage, assimilable à un « signe de la propriété ».

\section{Pour une histoire juridique de l’accès}

26. Tâchons maintenant de tirer quelques enseignements de cette leçon pour aborder la question des évolutions contemporaines des débats sur la propriété. Une première remarque concerne la difficulté de situer la possession dans la comparaison entre le modèle de la propriété divisée, propre à l'ancien Régime, et celui de la pleine propriété, c'est-à-dire du modèle exclusif de la propriété que les codifications napoléoniennes ont imposé à une partie de l'Europe et du monde. Dans la mesure où elle se distingue de la propriété, et à plus forte raison de la pleine propriété qui s'assimile à une sorte de petite souveraineté sur la chose, par où le propriétaire se voit disposer sur son bien d'un pouvoir semblable à celui de Dieu sur la création, la possession semblerait apte à désigner un rapport plus informel et provisoire à la chose, plus souple et plus plastique, qui sans avoir la force d'un titre juridique univoque et certain manifesterait un attachement de fait de l'homme à la chose et de la chose à l'homme, un rapport intime certes trop fragile pour constituer un droit à proprement parler, mais suffisamment solide pour être reconnu par le droit comme digne d'une protection provisoire. De fait, on a parlé d'une « autre façon de posséder » (un altro modo di possedere) pour décrire l'expérience juridique de l'Ancien Régime. Il s’agissait par là, comme l'écrit Paolo Grossi, d'opposer au «subjectivisme exaspérant» du législateur napoléonien « la vision plus complexe et plus harmonieuse d'un monde juridique dans lequel les choses n'aient pas pour seule vocation de s'offrir passivement à la volonté d'un sujet souvent arbitraire et déraisonnable $\gg^{56}$.

27. Pour autant la possession constitue-t-elle vraiment la clé d'accès à la bonne compréhension du régime féodal ? Les grandes lignes de ce régime sont connues. D’un côté, la situation du servage s'appuyait sur l'existence juridique de servitudes personnelles, qui en pratique imposaient aux paysans une position d'accessoires de la terre à laquelle leur service s’attachait. De l'autre côté, les divisions du dominium étaient si nombreuses qu'elles aboutissaient à ce qu'une série d'acteurs différents puissent revendiquer sur une

52 Si le corpus iuris n'en traite pas parmi les obligations, cela tient à l’approche procédurale des juristes de Rome, qui séparaient les interdits des obligations au motif que seules ces dernières donnent lieu à l'action ( $i b i d, p .50$; trad. fr. Faivre, p. 3I).

$53 R B, \S 39$, p. 4 II- $42 \mathrm{I}$.

54 Pour une reconstitution du développement de cette clausule, voir Luigi Labruna, Vim fieri veto. Alle radici di una ideologia, Camerino, Joveno, 1971, ch. 2, «Vis et tutela interdittale ».

55 Savigny cite divers auteurs qui ont soutenu cette opinion au XVII siècle, $R B, \S 6$, p. 54 ; trad. fr. Faivre, p. 35 .

56 «Una visione piu complessa ed armonica di un mondo giuridico nel quale non puó spettare alle cose il solo compito di oggetto passivo di volontà spesso arbitrarie e irragionevoli »(P. Grossi, « La proprietà e le proprietà nell'officina dello storico $\gg$, intervention au Congrès national des historiens du droit italiens, tenu à Sienne à l'automne 1985 , repris dans $i d$., Il dominio e le cose. Percezioni medievali e moderne dei diritti reali, Milan, Giuffrè, 1992, p. 603-675, p. 604. 
seule terre une série de droits différents. Comme le notait Édouard Meynial dans une étude classique sur les divisions médiévales de la propriété, « il y a bien des propriétaires d'une même chose, autant que l'organisation sociale comporte de cercles concentriques qui enserrent l'individu et autant qu'il y a de manières distinctes d'user ou de tirer profit d'une chose ${ }^{57}$. L'un dans l'autre ni les hommes n'étaient libres, ni les terres. Les premiers étaient toujours les vassaux d’autrui, les secondes étaient immanquablement grevées de quelque servitudes ${ }^{5}$. C'était là, encore, le type de situations juridiques auxquelles Savigny faisait face. Son programme politique, à bien des égards, consistait à faire sortir l'Allemagne de ce monde réputé « harmonieux », en ouvrant non seulement à l'abolition du servage, mais à la Bodenmobilisierung, c'est-à-dire au fait d'assurer l'accessibilité à la propriété foncière ou de faire en sorte que la terre puisse entrer dans le commerce à la manière d'une marchandise. De manière significative, la nomination de Savigny comme ministre de la Justice de l'État prussien coïncidera à peu près avec les premières réformes libérales du Landrecht. Pour autant, Savigny restait viscéralement opposé à la solution française d'une abolition législative des droits féodaux. La codification révolutionnaire - dont Merlin de Douai avait préparé le travail dans son rapport du 8 Février 1790, présenté à la Commission parlementaire pour l'abolition des droits féodaux - apparaissait à Savigny comme une aberration. Il n'hésitera pas, comme on sait, à y voir un « cancer $» 5$.

28. Au contraire, la relecture du droit romain de la possession devait préparer la sortie du système des droits féodaux. En refusant d'associer la possession au système des dominia médiévaux, en la déclarant un simple fait, en réhabilitant contre une longue tradition de commentaires savants ${ }^{60}$, l'idée romaine qu'elle ne pouvait avoir pour objet que des choses corporelles - n'étant elle-même qu'un fait protégé par égard pour l'intégrité des personnes, Savigny ôtait l'un de leurs fondements décisifs à l'ensemble de ces droits féodaux que le droit romano-canonique permettait de revendiquer à travers le système des actions possessoires. Faut-il en conclure que, chez Savigny, la possession devient tributaire de cette même « vision individualiste de la propriété » qu'on a dénoncée comme un mythe et comme une exaspération sacralisante des pouvoirs de l'individu ? Quoiqu'il en soit de l'idée de la propriété qu'a pu défendre Savigny, il est certain que son interprétation de la possession est ambivalente. Elle est l'arme technique qui doit permettre au professeur de droit d'influer indirectement sur le droit positif, au prétexte du droit romain, pour entailler le système des droits féodaux. Mais elle est aussi l'image d'un mélange de fait et de droit qui appuie sur la protection de la personnalité la défense de situations incommensurables à tout rapport de propriété. De fait, ceux qui opposent aujourd'hui le mythe moderne de la propriété à d'autres modes de posséder risquent peut-être d'accorder trop de solidité aux mythes. Faut-il vraiment renverser les principes de l'individualisme possessif, qui formerait le fond spéculatif de la philosophie de lappropriation de droit naturel ? Faut-il tenir le droit romain pour un instrument technique univoque, qui aurait permis le passage massif de cette philosophie dans le droit positif ? Une exploration attentive des archives de l'histoire du droit savant est plus que jamais nécessaire, pour rappeler l'ambivalence des

57 É. Meynial, « Notes sur la formation de la théorie du domaine divisé du XII au XIV siècles dans les Romanistes », dans Mélanges Fitting, t. II, Aalen/Francfort-sur-le-Main, Scientia, I969 [1908], p. 409-46I, p. 414, cité par R. Feenstra, « Les origines du dominium utile chez les glossateurs, avec un appendice concernant l'opinion des Ultramontani », dans Flores legum H. J. Scheltema oblati, R. Feenstra, J. H. A. Lokin, N. van der Wal (dir.), Groningen, Wolters-Noordhoff, 1971, p. 4993, p. 54 .

58 Que la terre n'ait pas été libre, c'est ce qu'indique a contrario l'article du Code rural révolutionnaire français de I79I : « Le territoire de France, dans toute son étendue, est libre comme les personnes qui l'habitent ». En Allemagne, ce fut tout l'enjeu de la « Bodenmobilisierung » ou de la «Befreiung des Bodens » (libération de la terre), que d'assurer l'accessibilité à la propriété foncière, c'est-à-dire de faire en sorte que la terre puisse entrer dans le commerce à la manière d'une marchandise. Pour une description du programme de l'école historique allemande rapportée au contexte de la question agraire, voir James Whitman, The Legacy of Roman Law in the German Romantic Era, Princeton University Press, 1990. Voir N. Habermann, «Die preussische Gesetzgebung zur Herstellung eines frei verfügbaren Grundeigenthums », dans Wissenschaft und Kodifikation des Privatrechts im I9. Jabrbundert, t. III, Die rechtlichte und wirtschaftliche Entwicklung des Grundeigentums und Grundkredits, H. Coing et W. Wilhelm (dir.), Francfort sur le Main, Klostermann, 1976, p. 3-43.

59 Sur l'emploi du mot en ce sens par Savigny, voir A. Dufour, Introduction à De la vocation, op. cit., p. 8.

6o Comme le rappelle Marta Madero, si le droit romain reconnaissait une quasi possessio, qui pouvait s'appliquer à certaines servitudes réelles, les canonistes médiévaux étendirent considérablement le domaine de cette exception au principe romain, selon lequel on ne peut posséder que des biens matériels. Innocent III par exemple reconnaît la possibilité de posséder une obéissance, un privilège d'immunité, un droit d'élection, un office ecclésiastique, etc. (M. Madero, « Penser la physique du pouvoir. La possession de la juridiction dans les commentaires d'Innocent IV et d'Antonio de Budrio à la décrétale Dilectus », Clio@Themis, II, 20I4, \$ I3, http://www.cliothemis.com/Penser-la-physique-du-pouvoir-La). 
catégories juridiques romaines, qui tout en ayant joué un rôle de premier plan dans le développement d'une dogmatique moderne, savante et systématique, n'ont sans doute pas vocation à être enrôlées trop vite dans les débats manichéens sur la propriété qui sont au cœur de l'idéologie des modernes. Une enquête circonstanciée de ce type importe sans doute, à l'heure où la pensée économique exerce sur l'évolution du droit une importance croissante, tandis que la réflexion sur les communs bat son plein ${ }^{61}$ et que l'accès fait figure de nouveau modèle de création de la valeur ${ }^{62}$.

\section{Pierre THÉVENIN}

6I En témoigne M. Cornu, J. Rochfeld et F. Orsi, Vocabulaire juridique des communs, Paris, Presses universitaires de France, 2017.

62 J. Rifkin, L'âge de l'accès. La nouvelle culture du capitalisme, Paris, La Découverte, 2005. 